\title{
Visual outcome and patient satisfaction after cataract surgery: A pragmatic study
}

\author{
Rashmi Mittal $^{\mathrm{a}}$, Jayanthi Peter ${ }^{\mathrm{a}, *}$, Thenmozhi Mani ${ }^{\mathrm{b}}$, Sarada David ${ }^{\mathrm{a}}$ \\ a Department of Ophthalmology, Christian Medical College, Vellore, India \\ ${ }^{\mathrm{b}}$ Department of Biostatistics, Christian Medical College, Vellore, India
}

\section{A R T I C L E I N F O}

\section{Keywords:}

Refractive outcome

Cataract surgery

Techniques

Vision

Patient satisfaction

\begin{abstract}
A B S T R A C T
Introduction: Cataract is a leading cause of blindness globally. This study compared the best corrected visual acuity (BCVA), surgically induced astigmatism (SIA), endothelial cell loss, and patient satisfaction between corneal section phacoemulsification (CSP) and Blumenthal manual small incision cataract surgery (MSICS).

Methods: In this pragmatic study, following pre-operative assessment, patients made a choice to undergo either CSP or MSICS after the options were explained by the ophthalmologist. Pre- and post-operative (Day 1, 1-week, 6-weeks) refraction, keratometry and specular microscopy were performed. Subjective improvement in visual function was assessed before surgery and at 6-weeks post-surgery using the 7-item VF-7 scale that assesses functional status and quality-of-life changes.

Results: Of the 178 eyes, 99 had CSP and 79 MSICS. At 6-weeks, BCVA of < 6/18 was achieved in 100\% in CSP and $96.2 \%$ in MSICS ( $\mathrm{p}=0.27$ ). The mean (SD) SIA was significantly lower ( $\mathrm{p}=0.003)$ with CSP $(1.29 \pm 0.71$ D) than MSICS $(1.01 \pm 0.49$ D). Endothelial loss was lower $(p=0.008)$ with MSICS (271 \pm 280 vs. $527 \pm 475$ cells $/ \mathrm{mm}^{2}$ ). Subjective improvement in visual function was better after MSICS than with CSP.

Conclusion: Despite similar visual acuity with CSP and MSICS, subjective improvement was better with MSICS. SIA was lower with CSP while endothelial loss was lower with MSICS.
\end{abstract}

\section{Introduction}

Impaired vision has a tangible impact on quality of life. Cataract is a leading cause of blindness in India. ${ }^{1}$ Although cataract surgery enables restoration of vision, there are barriers to providing this service to the millions who require it. ${ }^{2}$ Refinements in surgical procedure have enabled better visual outcome. Phacoemulsification and Blumenthal manual small incision cataract surgery (MSICS) are popular surgical techniques. The former, done using either ultrasound or laser, involves the implantation of a foldable intraocular lens (IOL) while MSICS commonly uses rigid IOL. Phacoemulsification has the advantage of smaller incision with less surgically induced astigmatism (SIA), but is machine dependent and costlier. ${ }^{3}$ MSICS, on the other hand, is safe, cost-effective and faster and can be used for all types of cataracts. ${ }^{4}$ Meta-analysis suggests that there is no difference in visual outcome between these techniques. ${ }^{3,5}$ In India where the cataract burden may influence the choice of technique, it is also important to look at qualitative factors such as visual outcome, patient satisfaction as well as cost. $^{6}$ This pragmatic study was thus undertaken to compare the best corrected visual acuity (BCVA), SIA, endothelial cell loss and patient satisfaction between corneal section phacoemulsification (CSP) and MSICS.

\section{Methods}

This study was done in a university affiliated teaching hospital over 9-months. All patients needing cataract surgery, following complete evaluation, were counselled on surgical options, expected visual outcome and cost. Once patients made their choice (CSP or MSICS), they were asked for their willingness to participate in this study. Exclusion criteria were pre-existing corneal pathology, hard cataracts, high astigmatism, increased axial length, need for concomitant ocular surgery and unwillingness to participate. The study was approved by the Institutional Review Board. Informed consent was obtained from the patient.

Objective and subjective refraction, automated keratometry, pachymetry and specular microscopy were done by a trained optometrist pre-operatively. A detailed slit-lamp examination was done by an ophthalmologist. Endothelial cell count and central corneal thickness were measured. Subjective assessment of visual function was assessed

\footnotetext{
* Corresponding author. Department of Ophthalmology, Christian Medical College, Vellore, 632 004, India.

E-mail addresses: rashmimittal2002@gmail.com (R. Mittal), jayanthi.peter@yahoo.com (J. Peter), saradadavid@gmail.com (S. David).
} 
using the VF-7 scale. ${ }^{7,8}$ Of the seven parameters in this scale, one parameter in the Finnish scale (driving at night) was replaced with an Australia VF-7 parameter (reading a newspaper or book) to adapt to our social setting. The SRK II formula was used to calculate IOL power. ${ }^{9}$

Participants underwent CSP or MSICS as per standard surgical protocols, by experienced surgeons who had performed at least 500 cataract surgeries. Patients were seen on Day 1, 1-week and 6-weeks. Objective and subjective refraction, automated keratometry, pachymetry and specular microscopy were repeated at 6-weeks by the same optometrist. Slit-lamp examination and VF-7 assessment were repeated at 6-weeks.

SIA was calculated from pre- and post-operative keratometry at 6weeks. BCVA, endothelial cell loss and changes in central corneal thickness (CCT) following surgery were recorded. Patient satisfaction was noted as the difference between pre-and post-operative VF-7 score. Statistical analysis was done using SPSS version 16. Categorical values were compared using chi-square; student's t-test was used to compare outcome differences between CSP and MSICS.

\section{Results}

Of the 178 patients recruited, 99 underwent CSP and 79 MSICS. Baseline characteristics are summarized in Table 1. The CSP arm had significantly $(\mathrm{p}<0.001)$ more soft cataracts than MSICS. All eyes in the MSICS group had rigid IOLs implanted whereas foldable IOL was used in all eyes undergoing CSP.

Over $90 \%$ of patients had an uncomplicated surgery. Intraoperative complications in CSP included posterior capsular rent $(n=2)$ and hazy cornea $(n=1)$. In the 2 patients with posterior capsule rent, IOL was placed in the ciliary sulcus. In the MSICS group, bleeding $(n=3)$, wound suturing $(n=2)$ and hazy cornea $(n=1)$ were encountered.

Table 1

Baseline characteristics of the patients.

\begin{tabular}{|c|c|c|}
\hline Characteristic & $\begin{array}{l}\text { Phacoemulsification } \\
(\mathrm{n}=99)\end{array}$ & $\begin{array}{l}\text { Manual small incision } \\
\text { cataract surgery } \\
(\mathrm{n}=79)\end{array}$ \\
\hline Age; Mean (SD) years & $59.8(9.7)$ & $62.7(9.7)$ \\
\hline Male: Female & 37: 62 & 35: 44 \\
\hline Operated eye (right: left) & 60: 39 & 53: 26 \\
\hline \multicolumn{3}{|l|}{ Grade of nucleus } \\
\hline $\begin{array}{l}\text { Nuclear sclerosis } \\
\text { Grade } 1\end{array}$ & $16(16.2)$ & $6(7.6)$ \\
\hline $\begin{array}{l}\text { Nuclear sclerosis } \\
\text { Grade } 2\end{array}$ & $60(60.6)$ & $35(44.3)$ \\
\hline $\begin{array}{l}\text { Nuclear sclerosis } \\
\text { Grade } 3\end{array}$ & $17(17.2)$ & $37(46.8)$ \\
\hline $\begin{array}{l}\text { Posterior subcapsular } \\
\text { cataract }\end{array}$ & $6(6.0)$ & $1(1.3)$ \\
\hline \multicolumn{3}{|l|}{ Status of the other eye } \\
\hline Cataract & $64(64.7)$ & $58(73.4)$ \\
\hline Pseudophakia & $28(28.3)$ & $165(19.0)$ \\
\hline $\begin{array}{l}\text { Pseudophakia with } \\
\text { PCO }\end{array}$ & $5(5.0)$ & $4(5.0)$ \\
\hline Clear lens & $1(1.0)$ & $1(1.3)$ \\
\hline Other pathology & $1(1.0)$ & $1(1.3)$ \\
\hline Diabetic & $36(36.4)$ & $19(24)$ \\
\hline \multicolumn{3}{|l|}{ Vision in the other eye } \\
\hline $6 / 6$ to $6 / 18$ & $50(50.5)$ & $26(33.3)$ \\
\hline $6 / 24$ to $6 / 60$ & $41(41.4)$ & $44(56.4)$ \\
\hline $5 / 60$ to $3 / 60$ & $5(5.1)$ & $7(9.0)$ \\
\hline Less than $3 / 60$ & $3(3.0)$ & $1(1.3)$ \\
\hline
\end{tabular}

SD - Standard deviation; values in parentheses indicate percentages unless indicated otherwise; PCO - posterior capsule opacification.
Table 2

Comparison of surgical induced astigmatism between phacoemulsification and minimal small incision cataract surgery.

\begin{tabular}{lllll}
\hline Surgical induced astigmatism & \multicolumn{2}{l}{ Phacoemulsification } & \multicolumn{2}{l}{ MSICS } \\
\cline { 2 - 5 } & $\mathrm{N}=99$ & $\%$ & $\mathrm{~N}=79$ & $\%$ \\
\hline$<0.5 \mathrm{D}$ & 14 & 14.1 & 11 & 13.9 \\
0.5 to $<1.00 \mathrm{D}$ & 38 & 38.4 & 10 & 24.1 \\
1 to $<2.00 \mathrm{D}$ & 44 & 44.5 & 34 & 43.0 \\
2 to $<3.00 \mathrm{D}$ & 2 & 2,0 & 14 & 17.7 \\
3 to $3.50 \mathrm{D}$ & 1 & 1.0 & 1 & 1.3 \\
\hline
\end{tabular}

MSICS - Minimal small incision cataract surgery; D - dioptres.

Late post-operative complications in CSP included posterior capsule opacification $(\mathrm{n}=2)$, corneal oedema, cystoid macular oedema and IOL decentration (one each). Following MSICS, late complications were posterior capsule opacification $(n=6)$, IOL decentration $(n=3)$ and optic capture $(n=2)$.

Post-operatively, overall $46.3 \%$ achieved an uncorrected visual acuity (UCVA) $6 / 12$ or better and $98.3 \%$ attained BCVA $6 / 12$ or better. More eyes that underwent CSP $(60.6 \%)$ had better UCVA $<6 / 18$ than MSICS (33.3\%). BCVA of $6 / 12$ or better was achieved in $100 \%$ and $96.2 \%$ of eyes following CSP and MSICS respectively. The mean spherical equivalent was $-0.26 \pm 0.56 \mathrm{D}$ following CSP and $-0.30 \pm 0.54 \mathrm{D}$ after MSICS. Three patients had best corrected near vision of J2 after CSP; one each due to foveal atrophy and amblyopia; no cause could be detected in the third patient. In the MSICS group three patients had posterior capsular opacity accounting for near vision of J5, J3 and J2. Other 5 eyes with J2 vision did not have any notable pathology.

The distribution of SIA after the two techniques is shown in Table 2. The mean (SD) SIA was significantly lower $(\mathrm{p}=0.003)$ with CSP $(1.29 \pm 0.71 \mathrm{D})$ than MSICS $(1.01 \pm 0.49 \mathrm{D})$. Only 2 patients in the CSP group had SIA of $\geq 2.0 \mathrm{D}$, compared with 15 in MSICS. SIA was more $(\mathrm{p}=0.09)$ in the left eye $(1.11 \pm 0.54 \mathrm{D})$ as compared to the right eye $(0.94 \pm 0.45 \mathrm{D})$. There was no effect of age on the SIA postoperatively.

Pre- and post-operative data on endothelial cell count was available for 73 eyes in CSP and 44 in the MSICS group. Endothelial loss was lower $(\mathrm{p}=0.008)$ with MSICS $\left(271 \pm 280\right.$ vs. $527 \pm 475$ cells $\left./ \mathrm{mm}^{2}\right)$. Endothelial cell loss was not significantly $(\mathrm{p}=0.22)$ different between diabetics and non-diabetics. CCT data was available in 96 patients with CSP and 77 patients with MSICS. In the CSP group, 54 patients had a gain in CCT post-operatively while 42 had a loss in CCT; in the MSICS group 41 had a gain while 36 had a loss in CCT. There was no difference in the change in the mean CCT pre- and post-operatively between the two groups $(\mathrm{p}=0.90)$.

Visual function score was calculated as the difference between the post and pre-operative visual function assessed by VF-7. Although the mean VF-7 score improved significantly ( $\mathrm{p}<0.001$ ) postoperatively following CSP (from 65.7 to 90.8) and MSICS (from 43.2 to 87.7), the average improvement in the visual function score was more dramatic (p $<0.001$ ) with MSICS (Fig. 1).

\section{Discussion}

In this pragmatic study involving 178 patients, BCVA following cataract surgery was similar with CSP and MSICS. Although the mean SIA was significantly lower with CSP than with MSICS and endothelial loss was lower with MSICS, subjective improvement in visual function was more dramatic following MISCS. These results have public health implications for developing countries that have a high burden of correctable blindness due to cataracts.

While cost-effectiveness may be the pivotal point in deciding the type of surgery to be implemented from a public health perspective, 


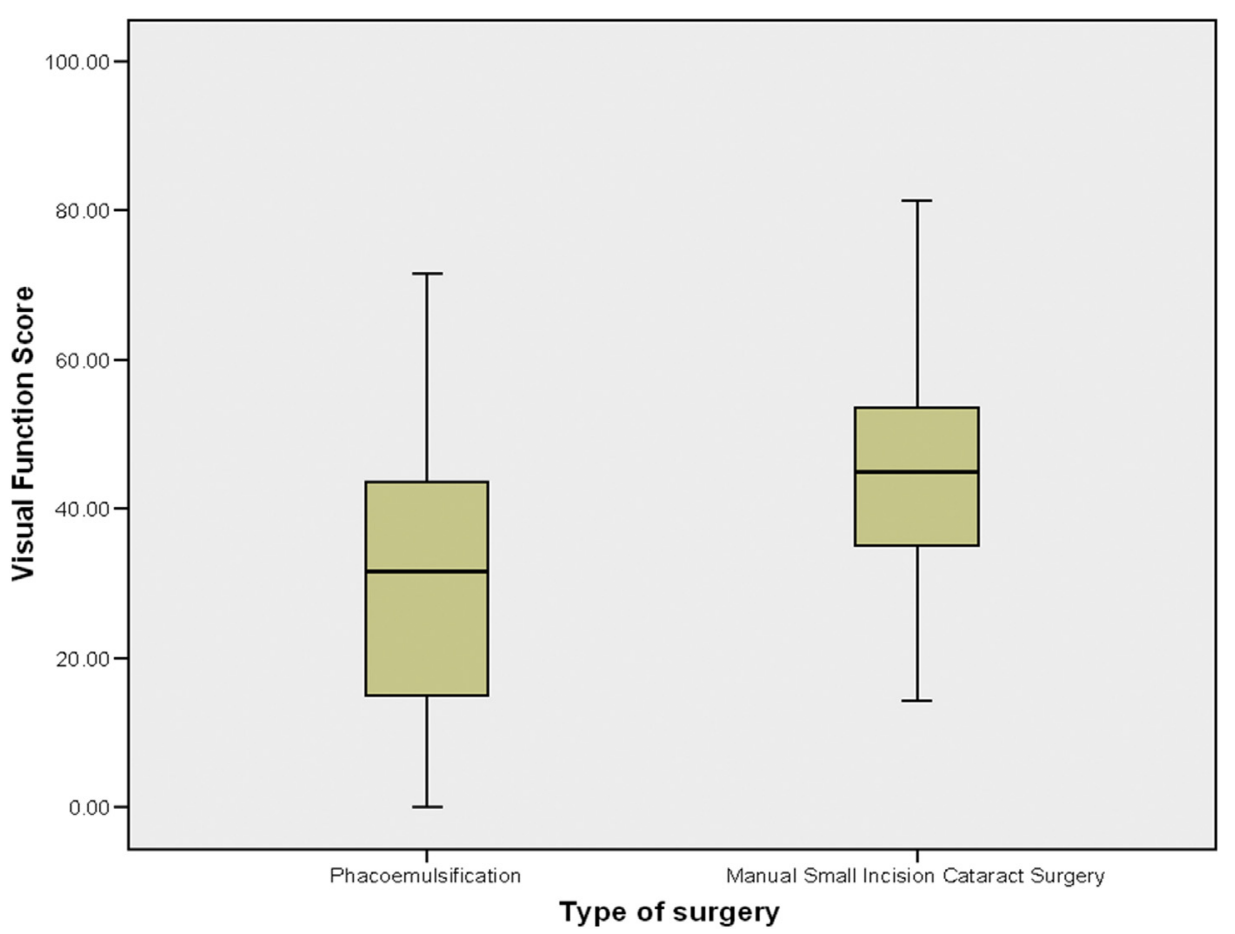

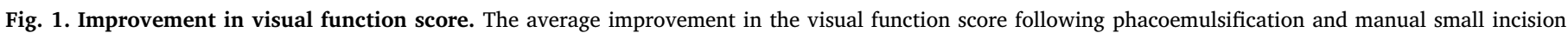
cataract surgery (MSICS) was more dramatic ( $<$ 0.001) with MSICS than with phacoemulsification.

objective and subjective measures of visual outcome may dominate decision making at an individual level. Meta-analysis of 11 studies has shown that visual outcomes are similar with both surgeries, consistent with the results of this study. ${ }^{3}$ Since MSICS may work out to be more cost-effective ${ }^{10}$ in view of lower capital investment cost, duration of surgery and type of lens used, it may be favoured for large volume surgeries such as eye camps. The use of the cheaper rigid lens in MSCIS without compromise in final BCVA when compared with the use of the more costly foldable lens in CSP in our study also supports the use of MSICS over CSP from a public health perspective. In the 3 studies that reported comparative costs of CSP and MSICS, MSICS was cheaper than CSP in all the 3 studies. ${ }^{11,12}$

It was surprising to note that the subjective improvement in visual function in our study, as assessed by the VF-7 score, was more dramatic with MSICS than with CSP. This could be explained by the fact that the VF-7 score was much lower pre-operatively in patients who underwent MSICS than with CSP. However it should be noted that the final VF-7 score was similar for MSICS $(87.7 \pm 12.5)$ and CSP $(90.6 \pm 10.8)$. One study from India that looked at improvement in subjective visual function following cataract surgery did not find a significant difference in VF improvement between MSICS and CSP. ${ }^{13}$ The fact that MSICS would be preferred over CSP for mature cataracts also tilts the balance in favour of MSICS for the vast majority.

As was observed in our study, SIA was less with CSP than with MSICS in other studies. ${ }^{3,14,15}$ A study by Kulkarni however did not show any significant difference in SIA between CSP and MSICS. ${ }^{13}$ Although BCVA was similar for CSP and MSICS in our study, more patients who underwent CSP had UCVA of $<6 / 18$. Endothelial loss in our patients was statistically significantly more with CSP than with MSICS; this loss did not appear to be clinically significant since the mean change in CCT pre- and post-operative was similar in both groups. The lower astigmatism and better UCVA with CSP may thus tilt patient preferences towards this procedure. However it should be noted that CSP is costlier, requires special equipment, additional training and skill and longer surgical time generally than MSICS.

The study must be interpreted in the light of the following limitations. Randomization was not possible because the choice of surgery was determined by the patient and governed by factors such as the stage of the cataract and socioeconomic status of the patient. The imbalance in VF-7 score at baseline between the two groups would have contributed to the more dramatic improvement in subjective score with MSICS. The fact that rigid lens was used in all patients undergoing MSICS and foldable lens in those who underwent CSP would have also impacted visual outcomes. Another limitation was that no attempt was made to correct pre-existing cylinder intra-operatively. This was not done in order to standardise the incision site for all patients. In clinical practice surgeons would decrease the post-operative astigmatism by intraoperative wound modulation.

Despite these limitations, the strength of the study is its pragmatic design that reflects day to day practice with choices made based on several factors. The study also demonstrated a more significant subjective improvement in visual function with MSICS when compared with CSP. Thus the results of this study are likely to be applicable to a larger population in India and in other developing countries.

In conclusion similar visual acuity was achieved with CSP and MSICS. SIA was lower with CSP. Although endothelial loss was lower with MSICS than CSP, this did not appear to be clinically significant reflecting in mean CCT post-operatively. The more dramatic subjective improvement in vision with MSICS and the lower cost makes it the surgical procedure of choice for cataracts in countries with a huge burden of visual disability due to cataract.

\section{Conflicts of interest}

There is no conflict of interest for any of the authors or any financial interests to disclose.

\section{References}

1. Bettadapura GS, Donthi K, Datti NP, Ranganath BG, Ramaswamy SB, Jayaram TS. Assessment of avoidable blindness using the rapid assessment of avoidable blindness methodology. N Am J Med Sci. 2012;4:389-393.

2. Finger RP. Cataracts in India: current situation, access and barriers in service over time. Ophthalmic Epidemiol. 2007;14:112-118.

3. Gogate P, Optom JJ, Deshpande S, Naidoo K. Meta- analysis to compare the safety 
and efficacy of manual small incision cataract surgery and phacoemulsification. Middle East Afr J Ophthalmol. 2015;22:362-369.

4. Thomas R, Kuriakose T, George R. Towards achieving small-incision cataract surgery 99.8\% of the time. Indian J Ophthalmol. 2000 Jun;48:145-151.

5. Riaz Y, de Silva SR, Evans JR. Manual small incision cataract surgery with posterior chamber lens versus phacoemulsification with posterior chamber intraocular lens for age related cataract. Cochrane Database Syst Rev. 2013;10 CD008813.

6. Gogate P, Deshpande M, Nirmalan P. Why do phacoemulsification? Manual small incision cataract surgery is almost as effective, but less expensive. Ophthalmology. 2007; 114:965-968.

7. Uusitalo RJ, Brans T, Pessi T, Tarkkanen A. Evaluating cataract surgery gains by assessing patients' quality of life using VF-7. J Cataract Refract Surg. 1999;25:989-994

8. Pager C Assessment of visual satisfaction and function after cataract surgery. $J$ Cataract Refract Surg. 2004;30:2510-2516.

9. Dang MS, Raj PP. SRK II formula in calculating intraocular lens power. $\mathrm{Br} J$ Ophthalmol. 1989;79:823-826.

10. Ruit $S$, Gurung R, Vyas $S$. The role of small incision suture-less cataract surgery in the developed world. Curr Opin Ophthalmol. 2018;29:105-109.

11. Gogate P, Deshpande M, Nirmalan P. Why do phacoemulsification? Manual small incision cataract surgery is almost as effective, but less expensive. Ophthalmology. 2007;114:965-968.

12. Khan A, Amitava AK, Rizvi SA, Siddiqui Z, Kumari N, Grover S. Cost effectivenes analysis should continually assess competing health care options especially in high volume environments like cataract surgery. Indian J Ophthalmol. 2015;63:496-500.

13. Kulkarni AN, Bhomaj P, Badhe G. Manual small incision cataract surgery is almost as effective but less expensive than phacoemulsification. Proceedings of the 73rd Annual Conference of the All India Ophthalmology Society 2010 at Kolkata, Cataract Session IV. 2010; 2010:146-148.

14. Ruit S, Tabin G, Chang D, et al. A prospective randomized clinical trial of phacoemulsification vs manual sutureless small-incision extracapsular cataract surgery in Nepal. Am J Ophthalmol. 2007;143:32-38.

15. Zhang J-Y, Feng Y-F, Cai J-Q. Phacoemulsification versus manual small-incision cataract surgery for age-related cataract: meta-analysis of randomized controlled trials. Clin Exp Ophthalmol. 2013;41:379-386. 